\title{
Spontaneous pseudomeningocele of sphenoid sinus or sphenoid mucocele?: A diagnosis dilemma
}

\author{
Noor Azrin Md Anuar ${ }^{1}$, Narizan Ariffin ${ }^{1}$, Teoh Jian Woei ${ }^{1}$, Balwant Singh Gendeh ${ }^{2}$ \\ ${ }^{1}$ Department of Otorhinolaryngology Head and Neck Surgery, Kuala Lumpur General Hospital, Malaysia \\ ${ }^{2}$ Department of Otorbinolaryngology Head and Neck Surgery, University Kebangsaan Malaysia Medical Centre \\ (UKMMC), Malaysia
}

\begin{abstract}
A pseudomeningocele is an abnormal collection of cerebrospinal fluid (CSF) that communicates with the CSF space around the brain or spinal cord. We describe a rare case of spontaneous non-traumatic pseudomeningocele in a 28 year-old gentleman who presented with delayed puberty associated with headache. Computed tomography revealed an expansile mass over bilateral sphenoid sinuses. Magnetic resonance imaging supported the diagnosis of sphenoid mucocele. A transnasal transphenoidal endoscopic sphenoidotomy was performed. Intraoperatively, despite an anatomical puncture through the sphenoid ostium, alarmingly, the opening leaked out CSF. A dehiscent was identified with a dural opening. This was repaired with multilayer technique. Clinical improvement was observed post-operatively.

The case reported here is unusual as they presented on imaging as a fluid collection in sphenoid sinus simulating a mucocoele, but were filled with CSF and represent pseudomeningocele. Surgeons should be alert to the presence of pseudomeningocele as they can mimic a mucocele or nasal polyp.
\end{abstract}

Key Words: CSF leak, mucocele, pseudomeningocele, transphenoidal surgery

\section{Introduction}

A pseudomeningocele is an abnormal collection of cerebrospinal fluid (CSF) that communicates with the CSF space around the brain or spinal cord. In a pseudomeningocele, the fluid has no surrounding membrane but it is contained in a cavity within the soft tissues, unlike meningocele, which is surrounded by dura layer. Any defects in the skull base can lead to spontaneous herniation of meningeal membranes and present as a meningocoele or meningoencephalocoele. The bony defect may be small and clinically silent until a breach in the meninges appears, leading to a cerebrospinal fluid (CSF) leak. In a spontaneous CSF leak, if the CSF remains contained by mucosa or soft tissue of the skull base, it will form a pseudomeningocele, a collection of CSF in the soft tissue that is not surrounded by arachnoid membranes. The typical presentations in the anterior cranial fossa include CSF rhinorrhea and a pulsatile or compressible nasal mass covered by attenuated meninges and mucosa. Although meningocoele, meningoencephalocoele, and spontaneous CSF leaks of the skull base have been recognized and are routine pathologies for the skull base surgeon and the rhinologist, spontaneous non-traumatic pseudomeningocele of the skull base has rarely been reported. The case reported here is unusual in that it presented on imaging as a fluid collection in sphenoid sinus simulating a mucocoele, but were filled with CSF and represent a pseudomeningocele.

\section{Case report}

In this literature we present a case of a 28 year-old gentleman who was referred to us from neuroendocrine team for further management of sphenoid mucocele. He had presented with history of delayed puberty with diminished facial, axillary and genitalia hair. $\mathrm{He}$ also complained of generalised intermittent episodes of headache for the past 2 years, which were throbbing in nature. The headache episodes were relieved temporarily with oral analgesia. Otherwise, he denied any nasal symptoms such as rhinorrhea, nasal congestion, or hyposmia. No symptom to suggest CSF rhinorrhea such as salty or sweet taste in the mouth. There was no eye symptoms such as blurring of vision, diplopia or eye proptosis, no otological symptoms, swallowing difficulties, loss of appetite or loss of weight. There was no history of head trauma or neurotology surgery.

*Corresponding Author: Dr. Noor Azrin Md Anuar, Department of Otorhinolaryngology Head and Neck Surgery, Kuala Lumpur General Hospital, Jalan Pahang, 50586, Kuala Lumpur. Malaysia, Tel: +6012-2407607, E-mail: miz.azrin@gmail.com 
General examination, revealed a young patient with diminished facial, axillary and pubic hair. There was no lymphadenopathy or cranial nerve palsy noted. The vision was normal with no evidence of proptosis. Nasal endoscopy examination with 0 degree rigid naso-endoscope was unremarkable.

A contrast-enhanced computed tomography (CECT) scan of paranasal sinus revealed the sphenoid sinus is expanded and completely occupied by hypodense lesion of fluid density with attenuation value of $13 \mathrm{HU}$ (Figure 1). There is scalloping and thinning of the wall of sphenoid sinus. Some part of the wall is dehiscence, involving the left lateral wall and posterior wall of the sphenoid sinus and the anterior wall of right carotid canal. There is dehiscence of the wall of cavernous internal carotid artery (ICA) bilaterally with the course of the left ICA is seen at the lateral aspect of the sphenoid sinus. However, both ICAs are patent. There is also dehiscence of the roof of the sphenoid sinus with the sella turcica with no clear demarcation between the pituitary gland and the lesion with evidence that sella is pushed superiorly by the lesion. The inferomedial wall of both optic canals are also dehiscence. Pneumatization is seen in both pterygoid body with likely dehiscence of vidian canal bilaterally. The wall of the sphenoid sinus bulged anteriorly into the posterior ethmoid air cells. Both sphenoid ostia are obliterated. However, the spheno-ethmoidal recesses are patent bilaterally. Otherwise, frontal, maxillary and ethmoid air cells are patent. The final impression of the radiological findings was in keeping with the sphenoid mucocele with dehiscence of its wall

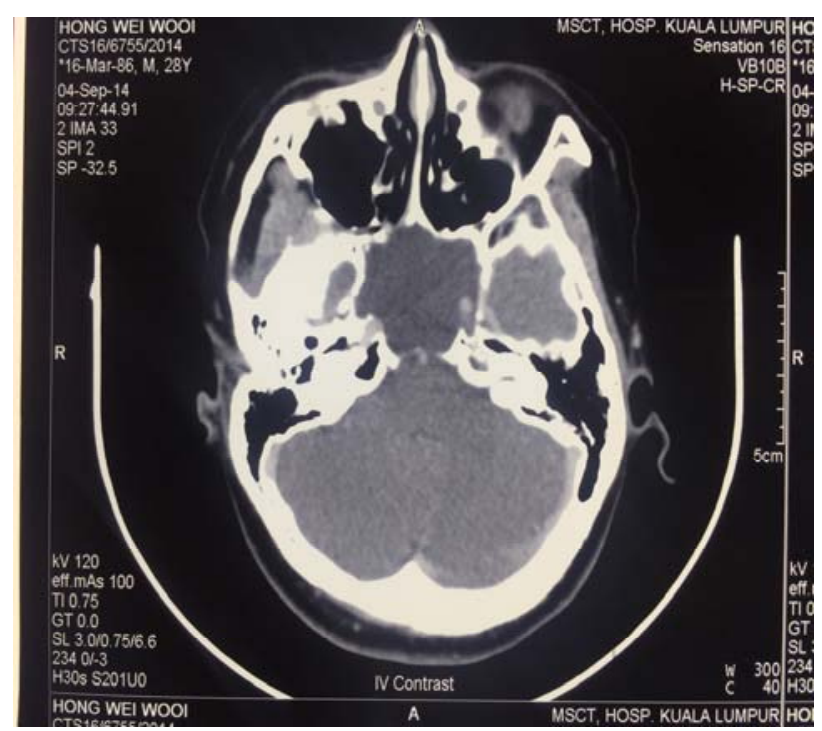

Fig. 1. CECT scan axial view. involving the optic canal, carotid artery, vidian canal, and floor of pituitary fossa.

A magnetic resonance imaging (MRI) scan was done and the findings was also suggestive of sphenoid mucocele with mass effect on the sella turcica and pituitary gland (Figure 2).

The patient was planned for transnasal transphenoid endoscopic marsupialization of sphenoid mucocele. Intraoperatively, bilateral sphenoidotomies were performed, but despite an anatomical puncture through the stenosed sphenoid ostium, alarmingly, the opening leaked out clear watery cerebrospinal fluid. There was significant expansion and destruction of bone of the sphenoid region, especially in the left lateral recess. On this side, the optic nerve and internal carotid artery exposure. A $2 \mathrm{~mm}$ breach in the dura with leakage of CSF was noted at the site where optic nerve is entering the dura posteriorly over the posterior wall of sphenoid sinus. A fat graft was plugged into the defect and fascia lata graft was placed over the dural defect to control the leak. Then nasal septal mucosal flap was transposed over the grafts at the left posterior sphenoid sinus wall. This was followed by tissue glue (Tissee1).

There was no evidence of cerebrospinal fluid (CSF) leak observed post-operatively. CSF fluid specimens were sent for biochemistry and C\&S examination. The biochemistry results confirmed the finding of CSF fluids.

At first month post-surgery follow-up, the patient had shown continual clinical improvement with no clinical evidence CSF leak and pituitary insufficiency.

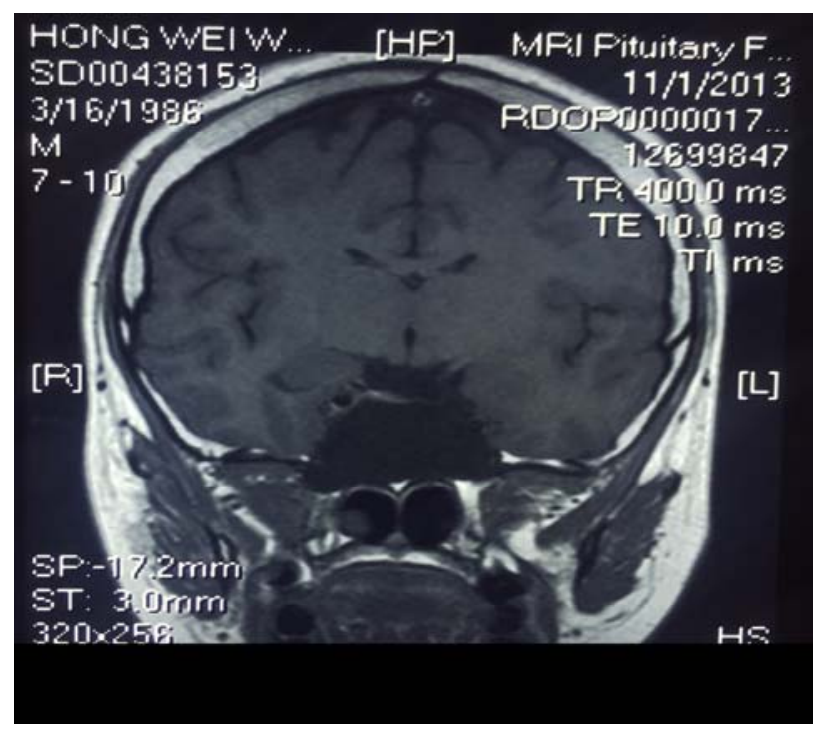

Fig. 2. MRI coronal view.

East J Med Volume:21, Number:3, July-September/2016 


\section{Discussion}

Spontaneous pseudomeningocele of the skull base is rare. Most reports of pseudomeningoceles published in past literatures are localized at the level of the thoracic or lumbar spine. The lesion is usually caused by an acute dural and arachnoid tear induced by surgery or trauma. Such tears occur most commonly at the junction of the theca with the nerve root sleeve, causing a pseudomeningocele in up to $2 \%$ of laminectomies (1). However, pseudomeningoceles rarely occur in the head and neck area. Again, trauma or iatrogenic injury was the primary cause in most of the reported cases in this region. For instance, CSF extravasation into the soft tissue occurs in $4.5 \%$ of neurotologic procedures (2) and is described as a rare complication of an atlantooccipital dislocation (3) or as a rare cause of posttraumatic orbital swelling (4). Here, we report, to our knowledge, a rare case of spontaneous pseudomeningocele affecting the skull base or the sphenoid sinus. While it is possible that pseudomeningoceles have been previously observed in the skull base or paranasal sinuses, and may even have been included in series of CSF leaks, the entity has not been recognized or reported as such.

In this case, CSF leaked through the ventral meninges at the skull base and accumulated in the local soft tissue and behind the mucosa of the sphenoid sinuses. Clinically, previous reported cases were associated with CSF rhinorrhea. But in the absence of CSF rhinorrhea, a pseudomeningocele may be difficult to differentiate from a mucocoele radiographically. To the best of our knowledge, only less than 20 cases of spontaneous idiopathic pseudomeningocele in the skull base region have been reported worldwide.

The pathophysiology of a pseudomeningocele involves both dural and arachnoid tears with leakage of CSF into the surrounding soft tissue, forming a fluid collection often surrounded by a fibrous capsule (4). We believe that pseudomeningoceles of the skull base originally develop like spontaneous CSF leaks. Small arachnoid tears lead to CSF leaks through small dural defects located at the skull base (5). The defect may be of developmental origin or a preceeding trauma. However, unlike most spontaneous CSF leaks where CSF extravasates directly into the sinus cavity, in spontaneous pseudomeningocele of the skull base, the CSF accumulates behind the mucosa of the paranasal sinus and may extend into the soft tissue of the infratemporal fossa or pterygopalatine space. In our patient there was no previous surgical trauma or history of blunt head trauma that may have played a role in the pathophysiology of the pseudomeningocele.

In the case reported here, the CSF leak responsible for the pseudomeningocele originated in the sphenoid sinus. Pseudomeningoceles may develop preferentially in the sphenoid sinus rather than the anterior ethmoid and cribriform regions because the dura overlying the sphenoid sinus may be less prone to herniation, and the mucosa of the sphenoid sinus more likely to be elevated without rupture. In pseudomeningocele, whether of spontaneous or post-traumatic origin, a small defect of the arachnoid and dura lets CSF leak into the diploic space via a small defect of the inner table (6). It is postulated that the hydrostatic pressure of the CSF trapped between the diploe increases via a ball-valve mechanism (6) or as a consequence of CSF pulsation (7), leading to bone resorption.

Our approach to treating pseudomeningoceles of the skull base is identical to the endoscopic repair of CSF leaks (8). Ideally, in preoperative preparation, a fine cut ( $1 \mathrm{~mm}$ or less) CT of the skull base to identify small erosions of the skull base. We also obtain an MRI to evaluate for an associated meningo-encephalocele. However, in this case CT scan and MRI revealed features suggestive of sphenoid mucocele and the diagnosis of pseudomeningocele was only made during intraoperative period. As an alternative if in doubt, a CT or MRI cysternography should be done prior to surgery to evaluate any CSF leak preoperatively to aid in diagnosis. Intraoperatively, we removed the pseudomeningocele and identified all the skull base and dural defects causing the CSF leak. This requires a wide exposure sphenoidectomy in order to facilitate the repair process.

Endoscopic surgery is the method of choice for repairing the majority of CSF leaks. It has minimal patient morbidity and the success rates are excellent at 76-97 percent (9). A recent metaanalysis estimates the success rate at the first attempt to be 90 percent (9). Many operative techniques and different types of graft material have been described (10). The grafts described include a nasal mucosal flap, free graft of nasal mucosa which may be a composite graft incorporating turbinate bone, conchal or septal cartilage, temporalis fascia and fascia lata which may be supported by fat (11-13). All of these 
techniques give a good success rate. All techniques require accurate localization of the leak intraoperatively. The graft material is then placed into the defect as an underlay graft where possible.

The patient should be advised not to blow their nose, to sneeze with their mouth open and not to stifle sneezes to avoid any abrupt increase in intracranial pressure, whilst they have an active leak and for a month postoperatively.

As a conclusion, Spontaneous Pseudomeningoceles of base of skull is extremely rare in the absence of trauma or iatrogenic injury. It occurs when CSF becomes trapped behind the paranasal sinus mucosa or extracranial soft tissues, and may be associated with bony erosion of the sphenoid bone or the clivus. Surgeons should be alert to their presence as they can mimic a mucocele or nasal polyp. Successful treatment can be obtained with endonasal endoscopic skull base surgical techniques. Increased recognition of this pathological entity is necessary to ensure proper management.

\section{References}

1. Smith DK, El-Sayed I, Pafundi E, Dolan RW. Presentation and treatment of a posttraumatic pseudomeningocele of the superior orbit. Am J Otolaryngol 2000; 21: 219-221.

2. Mehendale NH, Samy RN, Roland PS. Management of pseudomeningocele following neurotologic procedures. Otolaryngol Head Neck Surg 2004; 131: 253-262.

3. Goel A, Desai KI, Nadkarni TD, Muzumdar DP. An unusual post-traumatic occipitocervical pseudomeningocele: case report. Surg Neurol. 2001; 56: 62-65.

4. Mahapatra AK, Tandon PN. Post-traumatic intradiploic pseudomeningocele in children. Acta Neurochir (Wien) 1989; 100: 120-126.

5. Kaufman B, Nulsen FE, Weiss MH, et al. Acquired spontaneous, nontraumatic normalpressure cerebrospinal fluid fistulas originating from the middle fossa. Radiology 1977; 122: 379387.

6. D'Almeida AC, King RB. Intradiploic cerebrospinal fluid fistula. Report of two cases. J Neurosurg 1981; 54: 84-88.

7. Menku A, Koc RK, Tucer B, Akdemir H. Is skull fracture necessary for developing an intradiploic pseudomeningocele as a complication of head injury in adulthood? Acta Neurochir (Wien) 2004; 146: 623 .

8. Zweig JL, Carrau RL, Celin SE, et al. Endoscopic repair of cerebrospinal fluid leaks to the sinonasal tract: predictors of success. Otolaryngology Head Neck Surg 2000; 123: 195-201.

9. Hegazy HM, Carrau RL, Snyderman CH, Kassam A, Zweig J. Transnasal endoscopic repair of cerebrospinal fluid rhinorrhea: a meta-analysis. Laryngoscope 2000; 110: 1166-1172.

10. Hughes RGM, Jones NS, Robertson IJA. The endoscopic treatment of cerebrospinal fluid rhinorrhoea: The Nottingham experience. Journal of Laryngology and Otology 1997; 111: 125-128.

11. Snyderman CH, Kassam AB, Carrau R, Mintz A. Endoscopic Reconstruction of Cranial Base Defects following Endonasal Skull Base Surgery. Skull Base 2007; 17: 73-78.

12. Gendeh BS, Mazita A, Selladurai BM, et al. Endonasal endoscopic repair of anterior skull base fistulas: The Kuala Lumpur experience Journal of Laryngology and Otology 2005; 119 : 866-874.

13. Gendeh BS, Wormald PJ, Forer M, Goh BS, Misisran K. Endoscopic repair of spontaneous cerebro-spinal fluid rhinorrhea: A repair of 3 cases. Medical Journal of Malaysia 2002; 57: 503508. 